\title{
Reseña de Enrico Pattaro y Corrado Roversi (eds.), Legal philosophy in the Twentieth Century: The Civil Law World, Tome 1 "Language Areas" and Tome 2 "Main Orientations and Topics", Volume 12 of A Treatise of Legal Philosophy and General Jurisprudence, Springer, Dordrecht, 2017
}

\author{
Review of Enrico Pattaro y Corrado Roversi (eds.), Legal \\ philosophy in the Twentieth Century: The Civil Law World, \\ Tome 1 "Language Areas" and Tome 2 "Main Orientations \\ and Topics", Volume 12 of A Treatise of Legal Philosophy and \\ General Jurisprudence, Springer, Dordrecht, 2017
}

Federico José Arena*

Recepción: 19/02/2018

Evaluación: 26/02/2018

Recepción y aceptación final: 19/03/2018

\begin{abstract}
Resumen: El texto reseña brevemente los dos tomos que conforman el último Volumen del Treatise of Legal Philosophy and General Jurispruden$c e$, inicialmente editado por Enrico Pattaro y actualmente acompañado por Corrado Roversi. En la primera sección se reconstruye la estructura de la obra para luego, en las dos secciones siguientes, presentar sintéticamente el contenido de los diferentes capítulos que conforman el Tomo 1 y el Tomo 2, respectivamente. En cada caso se comenta con algo más de detenimiento las referencias a la filosofía del derecho en Latinoamérica, especialmente en Argentina.

Palabras clave: Filosofía del derecho, Civil Law, siglo 20, áreas lingüísticas, orientaciones, temas.
\end{abstract}

* Investigador Adjunto, CONICET (UNC) - Universidad Blas Pascal. Córdoba, Argentina.Email: fjarena@conicet.gov.ar 


\begin{abstract}
The text reviews briefly the two tomes belonging to the last Volume of the Treatise of Legal Philosophy and General Jurisprudence, initially edited by Enrico Pattaro and now with the participation of Corrado Roversi. In the first section, the text introduces the structure of both tomes and in the two following sections it offers an overview of Tome 1 and Tome 2, respectively. In each case the comments are focused on the references to legal philosophy in Latin America, primarly in Argentina. Keywords: Legal Philosophy, Civil Law, 20th century, language areas, orientations, topics.
\end{abstract}

\title{
I. Introducción
}

La obra que es objeto de esta recensión forma parte de un proyecto hercúleo que, si bien ambicioso, llega con éxito a su conclusión con este Volumen número 12, dedicado al mundo del civil law y dividido en dos tomos. Efectivamente, el Volumen 12 cierra el proyecto iniciado hace ya más de una década por Enrico Pattaro y titulado $A$ Treatise of Legal Philosophy and General Jurisprudence. Los volúmenes del Treatise fueron divididos en una primera parte teórica (que incluyó los primeros cinco volúmenes) y una segunda parte histórica (volúmenes seis a doce). Además, los editores señalan que se publicará un último volumen que contendrá un índice general. La envergadura de esta obra explica el epígrafe que abre el primer tomo aquí reseñado: "Nada está hecho mientras que no todo esté hecho" (cita de Pietro Nenni, Dari, 25 de julio de 1944).

Los doce volúmenes son ciertamente heterogéneos. Por un lado, algunos han sido completamente escritos por un único autor, mientras que otros han sido elaborados mediante el mecanismo de edición y colaboración de varios autores. Esto último es particularmente sobresaliente en el volumen doce, donde participan sesenta y tres autores. Por otro lado, la libertad concedida a los realizadores de cada volumen ha provocado cierta disparidad en la estructura y el lenguaje utilizado, como así también cierta incomunicación entre los volúmenes. Este problema ha sido resuelto en los últimos volúmenes y, sobre todo, en el 
volumen objeto de la presente recensión, ya que con bastante frecuencia se incluyen referencias al resto de los volúmenes. De todos modos, luego de leer el volumen objeto de la presente reseña, se advierte que esta pluralidad de puntos de vistas y de formas de abordar eventos históricos y problemas teóricos termina enriqueciendo la obra, ofreciendo distintas perspectivas.

Finalmente, también es necesario señalar que este último volumen es editado no sólo por Enrico Pattaro sino también por Corrado Roversi, quien fuera asistente de edición en el primer volumen. Ambos son académicos de la Universidad de Bologna, Italia.

\section{Estructura de la obra}

Este Volumen 12, junto al Volumen 11, están dedicados a la filosofía del derecho en el siglo 20. Mientras que el Volumen 11, obra de un único autor, a saber, Gerald Postema, se refiere al mundo del common law, el Volumen 12, obra colectiva, se ocupa, como señalé antes, del mundo del civil law. A diferencia del Volumen 11, el Volumen 12 está dividido en dos voluminosos tomos. Los editores explican esta decisión a partir del hecho que el mundo del civil law es en realidad una constelación de diferentes galaxias de autores e ideas, desparramados a lo largo y ancho del mundo. Por ello, intentando dar cuenta de esta variedad, se dividió el abordaje en dos partes. En el Tomo 1 la discusión está organizada por áreas lingüísticas. Este es el tomo más extenso, de 1062 páginas, y alberga contribuciones relativas a los países de habla germana (parte uno), a los países de Europa del sur y Francia (parte dos), a los países de Europa del este (parte tres), a los Países Bajos y al norte de Europa (parte cuatro) y a Latinoamérica (parte cinco). En este tomo cada una de las secciones fueron encargadas a autores que, o bien son nativos del idioma hablado en el país objeto de estudio, o bien son lo suficientemente hábiles en su manejo. E1 Tomo 2, en cambio, está organizado por orientaciones (partes una a tres) y temas (parte cuatro). Así, la primera parte, que contiene los primeros siete capítulos, está dedicada a la Teoría del Derecho natural. La segunda parte, capítulos 
ocho a once, al Positivismo jurídico. La tercera parte, que incluye del capítulo doce al capítulo veinte, está dedicada al Realismo jurídico. Finalmente, la parte cuatro se refiere al amplio tema del razonamiento jurídico. Entre ambos tomos, los países abordados son treinta y uno. En orden alfabético: Alemania, Argentina, Austria, Bélgica, Brasil, Bulgaria, Chile, Colombia, Croacia, Dinamarca, Eslovaquia, Eslovenia, España, Finlandia, Francia, Grecia, Holanda, Hungría, Italia, México, Noriega, Perú, Polonia, Portugal, República Checa, Rumania, Rusia, Serbia, Suecia, Uruguay y Venezuela.

De la breve descripción apenas hecha es posible advertir que la recensión de tamaña obra no resulta fácil. No sería posible comentar críticamente cada uno de los capítulos que constituyen los dos tomos de este último volumen del Treatise. No sólo por la extensión que tendría una reseña con esas ambiciones, sino, sobre todo, porque en virtud de la pluralidad y variedad de temas tratados es difícil hacer justicia al excelente trabajo de cada colaborador en una breve recensión. Por supuesto, todo ello, además, supera las capacidades del autor de esta reseña. En este sentido, las exigencias que los editores impusieron a los colaboradores, relativas al conocimiento de la lengua del país sobre el que escribían, se extienden indudablemente a la posibilidad de reseñar con mayor profundidad esos mismos capítulos. Por ello me limitaré aquí a agregar algunos comentarios adicionales relativos al tratamiento que, de la filosofía del derecho en Latinoamérica, se realiza en diferentes capítulos de ambos tomos. De este modo, respecto de cada tomo realizaré una breve presentación general y luego me referiré en particular sólo a algunos capítulos, concentrándome en aquellos referidos a Latinoamérica y, en especial, a Argentina. Esa elección no tiene, por supuesto, ninguna pretensión evaluativa acerca de la calidad del resto de los capítulos, sino que se debe a una selección arbitraria impulsada más que nada por la exigencia de brevedad y las limitaciones de quien escribe. 


\section{Tomo 1: Áreas lingüísticas}

Este tomo constituye una contribución fundamental para el estudio de la filosofía del derecho en los países del civil law. Así, los datos ofrecidos acerca de autores y corrientes teóricas son fundamentales para quien desee iniciar el análisis de la iusfilosofía en los países abordados. Pero además, y sobre todo, los autores de cada sección realizan un esfuerzo por combinar detalle y brevedad, de manera tal que es posible también identificar las tesis centrales y los respectivos desarrollos argumentales de las corrientes, movimientos o escuelas abordadas. Cabe señalar que, de manera poco común para obras en idioma inglés, la obra incluye también una detallada presentación de ideas y filósofos pertenecientes a países que no suelen formar parte de trabajos de este tipo. Así, además de los países europeos centrales, el tomo se refiere, no sólo a países de Latinoamérica, sino también de Europa del Este y a Rusia.

La primera parte del tomo está dedicada, como adelanté, a los países de lengua alemana. Se propone dividir el recorrido de esta área en dos eras, tomando como bisagra el año 1945. En efecto, los autores ven en este año un mojón para distinguir una primera era, "de las ideologías", que cubre las teorías y los autores de la primera mitad del siglo 20 y una segunda era, "del desencanto", que cubre la segunda mitad del siglo 20 y llega hasta nuestros días. Así, los capítulos 1 a 9, elaborados principalmente por Agostino Carrino, con la colaboración Giuliana Stella (sección 2.2 del capítulo 2 y capítulo 4) está dedicada a la era de las ideologías. En ella se analizan, primero, el resurgimiento del positivismo jurídico y el neo-kantismo. Luego el normativismo logicista, surgido alrededor de la teoría pura del derecho kelseniana. Por ello, además de la obra de Kelsen se analizan sus precursores: Frantisek Weyr y Hugo Krabbe, la escuela de Viena (Merkl y Verdross) y, por supuesto, los críticos de la teoría pura. En el capítulo 3 se aborda una de las alternativas al normativismo, a saber, la Filosofía del derecho sociológica. El capítulo 4 está dedicado a la Fenomenología del derecho. A continuación, en el capítulo 5 se aborda la crisis del neo-kantismo y el surgimiento del neo-hegelianismo, con especial detenimiento en su fundador Julius Binder. Este tratamiento de la filo- 
sofía del derecho alemana en la primera mitad del siglo es fundamental para leer luego los capítulos dedicados al positivismo jurídico incluidos en el Tomo 2, en particular el capítulo 8, dedicado al positivismo jurídico de la primera mitad del siglo 20 . Volviendo al Tomo 1, en los capítulos sucesivos (6 a 9) se presentan diferentes líneas de pensamiento: la revolución conservadora, el marxismo, el decisionismo y la filosofía del derecho del nacionalsocialismo. Tal como señalan los autores, estos primeros nueve capítulos dan cuenta de la riqueza (en el sentido de variedad) ideológica de la primera mitad del siglo 20 en los países de lengua alemana. El capítulo 10, último de esta primera parte y elaborado por Hasso Hofmann, está dedicado a la era "del desencanto" y toma como punto de partida lo que denomina "el shock de la injusticia de masas perpetrada por el derecho", para analizar las líneas de la filosofía del derecho alemana luego de 1945. Estas se caracterizan, de una parte, por el renacimiento de la escuela del derecho natural y el surgimiento de la filosofía del derecho de los valores de Radbruch. En igual sentido, en el Tomo 2, Francesco Viola se referirá a este fenómeno como al segundo revival del iusnaturalismo. De otra parte, la filosofía del derecho alemana luego de 1945 se caracteriza por la modernización de la teoría científica del derecho. Según Hofmann, esto se refleja en los trabajos de la teoría analítica del derecho y de la argumentación jurídica. Más adelante, el autor continúa con el análisis del efecto que tuvo la crisis del Estado de bienestar, produciendo un fenómeno de vuelta a la filosofía práctica y a la discusión de la idea de justicia. El capítulo se cierra con referencias a la globalización (la internacionalización del derecho constitucional y el universalismo de los derechos humanos) y con una retrospectiva de las teorías de la primera mitad del siglo 20 .

La segunda parte del Tomo 1 está dedicada a los países de Europa del sur y Francia. Así, el capítulo 11, autoría de Carla Faralli, aborda la filosofía del derecho en Italia y sigue una estructura histórica similar a la de la primera parte. Ello en cuanto comienza por describir el positivismo filosófico de fines del siglo 20 y su crisis, para luego analizar la reacción antipositivista que caracterizó al neo-kantismo y el neo-hegelianismo. A continuación, la autora se refiere a la caída del idealismo 
Reseña de Enrico Pattaro y Corrado Roversi (eds.)...

y a la situación de la postguerra, indicando las relaciones entre positivismo jurídico y filosofía analítica, la crisis del positivismo jurídico y las variantes post-positivistas más recientes. Esta estructura se refleja también en el tratamiento que del positivismo jurídico harán Mauro Barberis y Giorgio Bongiovanni en el Tomo 2. Para comprender la historia de la filosofía del derecho en Latinoamérica son fundamentales las páginas que Faralli dedica aquí a Norberto Bobbio, uno de los filósofos europeos más influyentes entre los autores latinoamericanos. En particular, tal como señala Faralli, su libro Scienza del diritto e analisi del linguaggio puede ser considerado como un programa de investigación que posteriormente atrajo a un gran número de filósofos. Demostrando la relevancia de Bobbio en el ámbito del civil law, a estas páginas de Faralli se suman varias referencias más al trabajo de ese autor, entre ellas, la de Barberis y Bongiovanni, en el capítulo 9 del Tomo 2, sobre la contribución metodológica de Bobio al debate iusfilosófico de postguerra y la de Pierluigi Chiassoni, en el capítulo 24 del mismo tomo, sobre el giro lingüístico propiciado por Bobbio.

El capítulo 12 está dedicado a Francia y, a diferencia del anterior, es obra de varios autores: André Jean Arnaud, Pierre Brunet, Vernique Champeil-Desplats, Jean Louis Halperin, Carlos Miguel Herrera y Eric Millard. Este capítulo también se diferencia estructuralmente de los capítulos dedicados al área alemana y a Italia, ya que en lugar de estar dividido por períodos históricos, está organizado por autores: Maurice Hauriou y su hipótesis institucional, Léon Duguit y la epistemología realista, Raymond Carré de Malberg y la teoría de las fuentes, las versiones de la escuela del derecho natural de François Gény y Michel Villey, y, finalmente, la teoría realista radical de la interpretación y del Estado de Michel Troper.

A España está dedicado el capítulo 13, escrito por Benjamín Rivaya. Si bien, al igual que los capítulos sobre el área alemana e Italia, Rivaya reconstruye la historia de la filosofía del derecho a partir de hitos históricos, en este caso se apoya en las vicisitudes de la sociedad española. Así, comienza por el monopolio del neotomismo en las primeras décadas del siglo 20, para abordar luego el pluralismo jurídico filosófico de los años de la República. A continuación, analiza 
la incidencia, primero, de la Guerra civil y, segundo, del franquismo en la filosofía del derecho española. Estas secciones terminan con un apartado dedicado a la caída de la filosofía del derecho franquista hacia mediados de los años setenta. El capítulo concluye con el recorrido de la filosofía del derecho durante la llamada transición hacia la democracia y la estabilización de esta última.

Portugal y Grecia, en los capítulos 14 y 15 respectivamente, cierran esta segunda parte dedicada a Europa del sur y Francia. El capítulo portugués está organizado asociando cada uno de los autores más importantes de la filosofía del derecho portuguesa a una línea filosófica y es obra de un grupo de autores: José Manuel Aroso Linhares, Luís Meneses do Vale, Alessandro Serpe y Ana Margarida Simões Gaudêncio y José de Sousa e Brito. El capítulo griego, en cambio, ha sido escrito por Constantinos Stamatis y retoma la organización de la primera parte, es decir, la bipartición en dos períodos: pre y post guerra, refiriéndose más extensamente al primero.

La tercera parte se ocupa de los países de Europa del Este. Quizás esta sea una de las contribuciones más novedosas de este volumen, ya que las historias de la filosofía del derecho suelen concentrarse en los países hasta ahora reseñados. Así, se presentan los recorridos de la filosofía del derecho en Polonia (capítulo 16), Rusia (capítulo 17), Checoslovaquia, primero y República Checa y Eslovaquia, después (capítulo 18, texto de Alexander Bröstl), Hungría (capítulo 19, texto de Csaba Varga) y, en el capítulo 20: Serbia (texto de Jasminka Hasanbegović), Croacia (texto de Ivan Padjen), Eslovenia (texto de Marijan Pavčnik), Bulgaria (texto de Vihren Bouzov) y Rumania (texto de Adrian-Paul Iliescu y Simina Tănăsescu).

El capítulo dedicado a Polonia (escrito por Tomasz Gizbert-Studnicki, Krzysztof Płeszka y Jan Woleński) está igualmente estructurado usando como eje la segunda guerra mundial, aunque en este caso se indaga con más detalle en el período de postguerra. El capítulo ruso (texto de Mikhail Antonov) está dividido en dos partes, el período prerrevolucionario y el período soviético y postsoviético. Si bien ambos capítulos, sobre todo el dedicado a Rusia, pueden parecer algo breves, ello queda compensado en el segundo tomo donde autores rusos y 
Reseña de Enrico Pattaro y Corrado Roversi (eds.)...

polacos son abordados en detalle, en especial, León Petrażycki y Jerzi Lande, maestro y discípulo, ambos nacidos en Polonia y profesores en la Universidad de San Petersburgo.

Llegamos así a la parte cuatro, dedicada a los países nórdicos y a los Países Bajos. El capítulo 20 se refiere a la filosofía del derecho sueca. El texto de Uta Bindreiter posee una estructura original, ya que propone identificar dos períodos luego de los años cuarenta: 19401960 y 1960-2000, según la influencia que ciertos filósofos o escuelas de pensamiento foráneos tuvieron en la filosofía del derecho sueca, distinguiendo a su vez, entre influencias fuertes y transitorias. Incluye además una interesante sección sobre el modo en que el trabajo de tres filósofos sentó las bases para el desarrollo de la filosofía del derecho como una disciplina académica autónoma en las Facultades de derecho suecas. Como puede advertirse, Bindreiter se concentra en el período de postguerra, a partir de los años cuarenta del siglo 20. Ello se debe, como se señala en la introducción del capítulo, a que toda la tercera parte del Tomo 2 está dedicada al Realismo jurídico, cuyos capítulos centrales se refieren a la Escuela de Uppsala, especialmente Axel Hägerström, Karl Olivecrona y Anders Vilhelm Lundstedt (escrito también por Bindreiter), entre otros.

Sigue luego el capítulo 22 donde se recorre la filosofía del derecho dinamarquesa. El lector debe complementar este capítulo con el dedicado a Alf Ross en el segundo volumen (capítulo 16). En efecto, Henrik Palmer Olsen, autor de este capítulo dedicado a Dinamarca, se refiere a la filosofía del derecho danesa antes y después de Ross, sin detenerse sobre este autor. Así, analiza en las primeras secciones las posiciones de Viggo Bentzon y Frederik Vinding Krusse, ambos maestros de Ross y también miembros, junto a Knud Berlin, del polémico tribunal de la Universidad de Copenhague que rechazó la tesis doctoral de Ross en $1927^{1}$. El capítulo se cierra con una sección en la que se analizan las propuestas críticas que, a partir de la hermenéutica, se ofrecieron como alternativa a la teoría de Ross.

1 Para los detalles de estos eventos véase la bien documentada biografía de Alf Ross escrita por Jens Evald en Evald, J., Alf Ross - a life, Tilst, DJOF, 2014, pág. 81 y ss. 
El capítulo 23 sobre Noruega y escrito por Svein Eng, el capítulo 24 sobre Finlandia y escrito por Susanna Lindroos-Hovinheimo, y el capítulo 25 sobre los Países Bajos (especialmente Holanda y la parte flamenca de Bélgica) y escrito a cuatro manos por Mark Van Hoecke y Arend Soeteman, siguen a grandes rasgos el esquema de la mayoría de los capítulos, dividiendo la periodización histórica en dos etapas, pre y postguerra, con el análisis de las distintas escuelas y/o tendencias surgidas durante esas épocas en cada uno de los países analizados.

Por último, la quinta parte está dedicada a la filosofía del derecho latinoamericana. En el capítulo 26, escrito por Manuel Atienza, se aborda el caso de la Argentina. Si bien es indudable que Atienza conoce con detalle la historia de la filosofía del derecho en Argentina, pues ha dedicado los primeros años de su investigación a su estudio ${ }^{2}$, se extraña la colaboración de un autor local para elaborar este capítulo, tal como sucede en la mayoría de los capítulos dedicados a áreas geográficas donde la filosofía del derecho ha tenido un desarrollo considerable. El autor se concentra en la segunda mitad del siglo 20 y distingue dos períodos tomando como punto de separación el año 1976, en que se produjo el golpe militar que inauguró la sangrienta dictadura argentina terminada recién con las elecciones de 1983. Esta estructura es común a varios otros capítulos sobre Latinoamérica. Es decir, a diferencia de los capítulos dedicados a países europeos, donde la Segunda guerra mundial produjo ciertamente efectos en el desarrollo de la filosofía del derecho, en Latinoamérica han sido los gobiernos dictatoriales los que han marcado, tanto en cuanto a contenidos como a los efectos en la vida de los académicos, el desarrollo de la filosofía del derecho. Ello es así en los casos de Brasil (dictadura 1964-1985) y Chile (dictadura pinochetista 1973-1990), por ejemplo. En el caso de Argentina, ello se evidencia, por un lado, en la persecución sufrida por numerosos filósofos que tuvieron que optar por el exilio, como Garzón Valdés y, por otro lado, en la incidencia que los juicios contra las juntas militares tuvieron en el desarrollo del neoconstitucionalismo. Tal es así que Atienza propone, con acierto, 
Reseña de Enrico Pattaro y Corrado Roversi (eds.)...

distinguir entre la filosofía del derecho producida en Argentina y la filosofía del derecho producida por argentinos.

El tratamiento de la filosofia del derecho en Argentina permite advertir lo que creo una virtud de este Volumen 12 del Treatise, aun cuando también conlleve ciertos riesgos. En efecto, ciertos temas se encuentran abordados en más de un capítulo o sección de ambos tomos del volumen. Si bien esto puede, a veces, producir cierta dispersión en la información, en realidad, creo, la pluralidad de perspectivas evita la mera repetición y, además, produce un efecto de entrelazamiento que termina por construir una trama de comprensión. Por ejemplo, y limitándonos sólo al tratamiento explícito, para apreciar la contribución que este volumen realiza al estudio de la filosofía del derecho en Argentina, es necesario combinar el capítulo escrito por Atienza con diferentes secciones del Tomo 2. Así, mientras Atienza hace un recorrido histórico de las distintas escuelas o corrientes de la filosofía del derecho rioplatense, en el Tomo 2 pueden encontrarse referencias a la misma zona geográfica y sus autores en numerosos puntos. En el capítulo 7, se hace referencia a la escuela del derecho natural en Argentina; en el capítulo 9, se aborda la contribución argentina al positivismo de postguerra; en los capítulos 1 (sección 1.4.5.2.) y 10, se presentan los desafíos al positivismo jurídico provenientes de las propuestas neoconstitucionalistas de Ernesto Garzón Valdés y Carlos Nino; en el capítulo 11, a su vez, se analizan las respuestas de Eugenio Bulygin y Jorge Rodríguez a ese mismo desafío neoconstitucionalista; en el capítulo 24, Chiassoni discute la teoría de la interpretación de Bulygin, entre otras referencias.

El capítulo 27, dedicado a la filosofía del derecho brasileña, es quizás uno de los más interesantes. Ya que, en razón del particular modo en que se desarrolló la filosofía del derecho en ese país, los autores hacen explícita una cuestión apremiante: qué cuenta como filosofía del derecho. Según los autores, en el contexto brasilero esa pregunta es relevante pues la historia de los estudios filosóficos en ese país tiene características que la distinguen de la mayoría de los países latinoamericanos, de habla hispana. Esos rasgos tienen que ver, sobre todo, con el rol que la denominada "misión francesa" tuvo en el desarrollo de la filosofía brasilera. Misión francesa es la expresión que los auto- 
res usan para referirse a un conjunto de intelectuales franceses que, a partir de la mitad de la década del treinta y en virtud de una política del Estado brasilero, llegaron a la Universidad de San Pablo e impusieron ciertas líneas y métodos de investigación ligados, sobre todo, al estructuralismo. Pero también, la historia de la filosofía del derecho en Brasil se caracteriza por una duradera falta de interés de los filósofos en el trabajo de los juristas (filósofos) y viceversa. Quizás consecuencia de ello sea que, salvo en los últimos años, la filosofía del derecho fue desarrollada por juristas dentro de ciertas disciplinas dogmáticas como el derecho administrativo y el derecho tributario. En definitiva, y a pesar de la existencia de algunos ejemplos de filósofos del derecho que se destacaron, como Miguel Reale, los autores se preocupan por la falta de una filosofía del derecho propiamente brasilera en un contexto donde la globalización de la cultura académica tiende a diluir estas construcciones. Este capítulo, en el entramado del volumen 12, debe ser completado con la breve referencia a la teoría del derecho natural en Brasil, que propone Carlos Massini Correas en la sección 3 del capítulo 7 del Tomo 2.

Cierra el tomo el capítulo 28, autoría de Rodolfo Vázquez, y en el que se abordan los desarrollos de la filosofía del derecho en Colombia, Chile, Costa Rica, México, Perú, Uruguay y Venezuela. Respecto de Chile, se señala algo que es efectivamente admitido por los mismos miembros de la comunidad iusfilosófica de ese país, es decir, la dependencia del pensamiento jurídico de las contribuciones europeas y anglosajonas ${ }^{3}$. De todos modos, cabría agregar a la reseña de Vázquez una referencia al papel que tuvo y tiene la Sociedad Chilena de Filosofía Jurídica y Social. El Anuario de la Sociedad, publicado desde 1983, ha contribuido al surgimiento en ese país de una comunidad iusfilosófica dispuesta al diálogo y con potencialidades para desarrollar autónomamente distintas líneas de pensamiento. Creo que también es dable señalar la influencia que las políticas gubernamentales de los últimos años, dirigidas a fomentar la investigación, han tenido en la

3 Véase Benavente, J.W., "La filosofía del derecho en Chile", Anuario de Filosofia Jurídica y Social, 1, 1983, pp. 21-39. 
reciente expansión de la filosofía del derecho en Chile. Así, los programas de becas para estudios de doctorados en el extranjero, más los incentivos para atraer académicos hacia las Universidades no metropolitanas, es decir Universidades de regiones fuera de Santiago, como la Universidad Austral, la Universidad de Temuco o la misma de Valparaíso, han contribuido a la formación y llegada de nuevos académicos $\mathrm{y}$ ha producido un aumento en los intercambios, tales como congresos internacionales y publicaciones periódicas.

Este último comentario me permite introducir una observación crítica referida a una omisión en las reconstrucciones históricas de la filosofía del derecho en Latinoamérica (aunque también está ausente en el tratamiento del resto de las áreas lingüísticas), a saber, la mención de un tipo de intercambio entre filósofos que ha adquirido gran relevancia en los últimos años: los congresos y reuniones científicas. Algunas de ellas han contribuido de manera decisiva a la circulación de las ideas en el ámbito continental. En Europa me parece fundamental el Seminario ítalo-español de Teoría del derecho, realizado por primera vez en la ciudad italiana de Imperia a mediados de los años noventa y que luego sumó, primero, a universidades francesas y, en los últimos años, a universidades portuguesas. Este seminario contribuyó, sin lugar a dudas, al intercambio entre académicos de Latinoamérica y Europa continental. De igual modo, en Latinoamérica parece decisivo el Seminario Internacional de Filosofía del derecho, conocido como Vaquerías, dirigido por Ricardo Caracciolo desde hace más de dos décadas. Este seminario, además de proponer a Córdoba como centro de reuniones científicas en filosofía del derecho, resultó un punto de intercambio similar al Seminario ítalo-español. En las últimas décadas este tipo de encuentros ha visto aumentar su número y desempeñan un papel central en el modo actual de hacer filosofía del derecho.

\section{Tomo dos: principales orientaciones y temas}

Este segundo tomo, como señalé más arriba, está organizado por orientaciones (partes una a tres) y temas (parte cuatro). Así, la primera parte, 
que contiene los primeros siete capítulos, está dedicada a la Teoría del Derecho natural. En la segunda parte, capítulos 8 a 11, se analiza el Positivismo jurídico. La tercera parte, que incluye del capítulo 12 al capítulo 20, aborda el Realismo jurídico. Finalmente, la cuarta parte está dedicada al amplio tema del razonamiento jurídico. Una virtud de este segundo tomo es que las ideas iusfilosóficas se conectan con sus bases o puntos de partida más filosóficos en general.

Esta diferencia en la estructura, respecto del Tomo 1, enriquece la contribución que el volumen hace al estudio de la cultura jurídica del civil law. Ello en cuanto el lector puede complementar el abordaje, mayoritariamente histórico-geográfico, del Tomo 1, con el abordaje mayormente por orientaciones y temas del Tomo 2, y construir así una suerte de trama de comprensión que permite capturar mejor la contribución de la cultura del civil law a la filosofía del derecho durante el siglo 20. Por ejemplo, los capítulos dedicados a la filosofía del derecho en países de lengua alemana se entrelazan con numerosos capítulos del Tomo 2. El capítulo 1 dedicado a la escuela del derecho natural, el capítulo 2 dedicado, particularmente, al desarrollo de la escuela de derecho natural en Alemania, los capítulos dedicados al positivismo jurídico (especialmente los capítulos 7 y 8 ) y el capítulo 25 sobre razonamiento jurídico que, entre otros, se refiere a la teoría de Jürgen Habermas y Robert Alexy. Entrelazando de este modo las contribuciones de ambos tomos es posible, como decía, construir una trama histórico-analítica para comprender la cultura filosófico jurídica del civil law. De todos modos, cabe señalar que algunos de los capítulos de este segundo tomo están construidos de manera demasiado análoga a los capítulos del primero; retomando, en algunos casos, la presentación histórico-cronológica, por área geográfica, en lugar de una presentación por orientaciones y temas. Creo que ello es lo que sucede con el capítulo 4 dedicado a la Escuela del derecho natural en Portugal y España y con el capítulo 7 dedicado a la Escuela del derecho natural en Latinoamérica. Algo parecido sucede con algunos capítulos de la sección dedicada al realismo jurídico, donde se retoma la clasificación por área geográfica. 
Reseña de Enrico Pattaro y Corrado Roversi (eds.)...

La primera parte del Tomo 2 está entonces dedicada a la Teoría del derecho natural. Comienza con un capítulo general, escrito por Francesco Viola, estructurado siguiendo lo que el autor considera los tres revivals del iusnaturalismo (intercalando, además, una mención a las relaciones entre iusnaturalismo y totalitarismo). Según el autor, el primer revival se produjo al iniciar el siglo 20, provocado por una insatisfacción con el positivismo jurídico decimonónico, reo de separar de manera demasiado estricta la esfera legal de las ideas morales y las bases sociales del derecho. El segundo, tuvo lugar luego de la Segunda guerra mundial, y fue originado por las violaciones a la dignidad humana durante esa conflagración, frente a las cuales el derecho positivo no pareció constituir un muro de contención. Y el tercero, que se desarrolla hacia el final del siglo 20 , fue provocado por la relevancia adquirida por los derechos humanos y la constitucionalización de los ordenamientos jurídicos, todo lo cual produjo una modificación en el modo de concebir el derecho positivo. Cabe señalar que revival no significa en todos los casos que se produjera un regreso a o resurgimiento de la Teoría del derecho natural como un todo, sino que en algunos casos se trata más bien, tal como lo indica Viola, de la incidencia que, en la discusión iusfilosófica, tuvieron tesis o inclinaciones que pueden ser asociadas con el iusnaturalismo.

Luego le siguen seis capítulos que analizan la evolución del iusnaturalismo en distintas áreas geográficas: Alemania en el capítulo 2, escrito por Stephan Kirste; Francia en el capítulo 3, autoría de Stamatios Tzitzis y Guillaume Bernard; España y Portugal en el capítulo 4, texto de Antonio-Enrique Pérez Luño; Italia en el capítulo 5, autoría también de Francesco Viola; Hungría en el capítulo 6, texto de Máté Paksy y Csaba Varga y América Latina en el capítulo 7, escrito por Carlos I. Massini Correas.

En estos capítulos, pero también en aquellos referidos al iuspositivismo y al iusrealismo, se evidencia la dificultad de separar el tratamiento de la discusión teórica en el ámbito del civil law de aquella llevada a cabo en el ámbito del common law. Efectivamente, a nivel de filosofía del derecho, la comunicación entre ambas áreas y, además, la influencia de autores continentales entre filósofos anglosajones, 
sobre todo en la primera mitad del siglo, y viceversa, sobre todo en la segunda mitad del siglo, exige introducir referencias cruzadas respecto de las tres orientaciones analizadas. Así, es inevitable mencionar en estos tomos también, por ejemplo, a John Finnis en el caso de la teoría del derecho natural, a Herbert Hart en el caso del iuspositivismo, o Ronald Dworkin para el anti-positivismo o neoconstitucionalismo, entre otros. De todos modos, los autores de los diferentes capítulos logran construir un análisis autónomo respecto del trabajo realizado por Gerald Postema en el volumen precedente, dedicado, como ya se dijo, a la filosofía del derecho del common law.

La segunda parte está dedicada al positivismo jurídico y, si bien presenta la evolución de esta corriente teórica siguiendo un recorrido histórico, creo que tiene el mérito de diferenciarse del Tomo 1 en cuanto no está estructurada en virtud de áreas geográficas, evitando el riesgo de solapamiento y agregando nuevas perspectivas y mayor densidad a la trama global del volumen. Los autores de esta segunda parte, Mauro Barberis (introducción y capítulos 9, 10 y 11) y Giorgio Bongiovanni (capítulos 8, 9 y 10), dividen el tratamiento del positivismo jurídico en distintas etapas. Comienzan por el positivismo jurídico de la primera mitad del siglo 20, indagando principalmente en las influencias neo-kantianas, presentando la teoría pura de Kelsen y la influencia de la Escuela de Viena. Los autores extienden el análisis llevado adelante en estos primeros capítulos a la teoría del derecho y del Estado desarrolladas en Francia, al debate entre derecho y política en Alemania, incluyendo el institucionalismo de Santi Romano y la deriva totalitaria de la filosofía del derecho en el corporativismo italiano. Siguen luego con el papel del positivismo jurídico en el debate de postguerra. Se detienen en especial en el argumento de Radbruch y las tesis del último Kelsen. Abordando también las contribuciones italianas, francesas, argentinas y españolas a ese debate.

Continúan luego con el desafío neoconstitucionalista y analizan la forma que a ese desafío han dado diferentes autores, entre ellos Ernesto Garzón Valdés, Carlos Nino, Robert Alexy y Luigi Ferrajoli, entre otros. Concluyen esta parte con una referencia a las respuestas que el positivismo jurídico ha ofrecido al desafío neo constitucionalista, en 
Reseña de Enrico Pattaro y Corrado Roversi (eds.)...

particular los intentos de Eugenio Bulygin, José Juan Moreso, Juan Carlos Bayón y Jorge Rodríguez, entre otros.

La tercera parte está dedicada al realismo jurídico, teoría que recibe el tratamiento más extenso dentro del tomo. Esta parte comienza con un capítulo introductorio de Edoardo Fittipaldi titulado "El realismo jurídico continental" que busca echar luz sobre el término "realismo" y las principales tesis que pueden ser atribuidas a esa escuela o movimiento. Los capítulos siguientes están organizados a partir de la obra de los autores principales dentro de este movimiento. Así el capítulo 13, escrito por Enrico Pattaro, analiza la obra de Axel Hägerstrom; el capítulo 14, autoría de Torben Spaak, se refiere a Karl Olivecrona; el capítulo 15 está dedicado a Anders Vilhelm Lundstedt y ha sido elaborado por Uta Bindreiter; el capítulo 16 analiza la obra de Alf Ross, y es autoría del Mauro Zamboni; y el capítulo 17 rescata otros realistas jurídicos escandinavos, por lo común poco mencionados en los debates contemporáneos, y es autoría de Uta Bindreiter, Mauro Zamboni y Torben Spaak. Del capítulo 18 al capítulo 20, Fittipaldi se refiere a los vínculos entre el realismo jurídico escandinavo y el realismo jurídico ruso-polaco. En un gran esfuerzo de presentación, en virtud de la dificultad de encontrar los textos originales, Fittipaldi presenta extensamente las propuestas teóricas de León Petrazycki (capítulo 18) y Jerzy Lande (capítulo 19). Asimismo, Elena V. Timoshina, Giuseppe Lorini y Wojciech Zetaniec analizan el trabajo de otros realistas jurídicos ruso-polacos en el capítulo 20. Se extraña en ese tratamiento del realismo jurídico, particularmente de Hagerstrôm y Olivecrona, un apartado sobre el rendimiento de sus teorías respecto de la discusión contemporánea.

La parte cuarta, que cierra el Tomo 2, deja entonces atrás la estructura por orientaciones y se dedica a analizar los temas de la filosofía del derecho continental del siglo 20. Algo ciertamente ya realizado, si bien de manera oblicua, en el Tomo 1 y en las primeras tres partes del Tomo 2. Es por ello que, como señalé, a partir de estas tres líneas de presentación (áreas geográficas, orientaciones y temas), es posible construir un interesante entramado para comprender la cultura jurídico-filosófica en el ámbito del civil law a lo largo del siglo 20. Esta cuarta 
parte, en manos principalmente de Pierluigi Chiassoni (capítulos 21 a 24) y Eveline Feteris (capítulo 23 y 25), cuenta también con la participación de Hanna Maria Kreuzbauer en el capítulo 23, Jan Wolenski, autor del capítulo 26 y de Davide Grossi y Antonio Rotolo, autores del capítulo 27 que cierra el Tomo, el Volumen y el Tratado.

El capítulo 21, titulado "La herencia del siglo XIX: la era del cognitivismo interpretativista" está dedicado a presentar el punto de partida de la filosofía del derecho del siglo 20 y aborda la escuela de la exégesis, la hermenéutica jurídica de Savigny y se cierra con una referencia a la denominada "jurisprudencia de conceptos" de von Jhering y Windscheid. En esta sección Chiassoni presenta de una manera "no ortodoxa" la Escuela de la exégesis. Según Chiassoni, el punto de vista tradicional caracteriza a la Escuela de la exégesis sobre la base de dos métodos interpretativos: el lenguaje literal y la intención del legislador tal como puede ser recabada de los trabajos preparatorios. Sin embargo, Chiassoni sostiene, por un lado, que las herramientas interpretativas usadas y propuestas por esta escuela son mucho más amplias y, por otro lado, que no es posible atribuir a sus miembros una posición unitaria acerca de los métodos interpretativos.

El capítulo 22, titulado "La era del descontento: la revuelta contra el interpretativismo cognitivista", aborda las reacciones a la jurisprudencia de conceptos en la forma de la crítica metodológica de Fançois Gény, el movimiento del derecho libre, la jurisprudencia de intereses y la teoría pura del derecho. El capítulo 23 se propone como un balance de temas, indagando en el redescubrimiento de la retórica, la argumentación por tópicos, la interpretación jurídica y la hermenéutica. El capítulo 24, que cierra la participación de Chiassoni, se titula "La era del análisis: empirismo lógico, lenguaje ordinario y la pura verdad sobre la cuestión". Allí el autor presenta lo que denomina el encanto del positivismo jurídico, las herramientas analíticas y la contribución al respecto del realismo jurídico de Ross y Tarello.

El capítulo 25, obra de Eveline Feteris, está dedicado a las contribuciones que diferentes autores realizaron al análisis de la argumentación jurídica. Así, se refiere a la teoría institucional de Neil MacCormick (una inclusión excepcional en un Volumen sobre civil law), a la teo- 
ría del discurso de Jürgen Habermas, a la teoría de la pretensión de corrección del derecho de Robert Alexy, a la teoría de la justificación de Aarnio y a la teoría del razonamiento judicial de Peczenik. El capítulo concluye con una sección sobre la teoría pragma-dialéctica de la argumentación jurídica.

El capítulo 26, de Jan Wolenski, aborda la intrincada relación entre derecho y lógica a lo largo del siglo 20, pasando por el conocido dilema de Jörgensen y los intentos por construir una lógica deóntica, hasta la relación entre lógica y argumentación jurídica. Por último, cierra el Tomo, Volumen y Tratado el capítulo 27, de Davide Grossi y Antonio Rotolo, dedicado a los más recientes desarrollos de la lógica jurídica. En particular se refieren a la lógica de las obligaciones, a la teoría de los sistemas normativos, a la incidencia de la derrotabilidad en el razonamiento jurídico y, por último, a la dinámica jurídica.

\section{Breves comentarios sobre el análisis de tesis de autores argentinos}

Tal como destaca Atienza, es posible encontrar en la filosofía del derecho Argentina del siglo 20 una gran variedad de corrientes. En el segundo tomo se encuentran analizadas teóricamente sólo dos de ellas, a saber, el positivismo jurídico y el neoconstitucionalismo. Es cierto, también hay un capítulo dedicado a la Teoría del derecho natural en Argentina, pero en esas páginas Massini Correas se concentra en proponer una cronología de los autores representantes de esa escuela, presentando, sin discutir desde un punto de vista teórico sus ideas. En cambio, en la segunda parte dedicada al positivismo jurídico, Barberis y Bongiovanni dedican varios párrafos a presentar y discutir, si bien brevemente, las posiciones de los filósofos argentinos, en particular, las de Carlos Alchourrón, Eugenio Bulygin, Ricardo Caracciolo, Ernesto Garzón Valdez, Caros Nino y Jorge Rodríguez, entre otros. Asimismo, Pierluigi Chiassoni presenta y analiza la posición de Bulygin respecto de la interpretación. A ello cabe agregar la referencia que a la posición de Carlos Nino hace Viola discutiendo el tercer revival del derecho 
natural, y atribuyendole cierta superposición con esa teoría. Finalmente, hay una breve referencia al trabajo de Alchourrón y Bulygin en el último capítulo, dedicado a derecho y lógica.

Tal como señala Atienza y refrendan Barberis y Bongiovanni, la filosofía analítica del derecho tuvo gran influencia en Argentina e incluso, creo posible afirmar, se produjeron contribuciones originales. Central en ese desarrollo fue Ambrosio Gioja y el Instituto de Filosofía del derecho de la Universidad de Buenos Aires. Allí surgieron los iniciadores de esa corriente en Argentina entre ellos Genaro Carrió, Carlos Alchourrón y Eugenio Bulygin. Si bien con matices, los tres defendieron versiones del positivismo jurídico. Por ejemplo, Carrió fue claramente defensor de una versión del positivismo que más tarde fuera denominada como incorporacionismo o positivismo incluyente. En efecto, Carrió fue uno de los primeros filósofos en ensayar una respuesta a las críticas que Dworkin formulara contra el positivismo en Los derechos en serio. Según una de esas críticas, la tesis hartiana de la existencia de una regla de reconocimiento, i.e., de una práctica regular entre los jueces de identificar las normas jurídicas a partir de ciertos criterios, no es capaz de capturar un tipo de norma jurídica, cuyo papel es esencial den la práctica jurídica, a saber, los principios jurídicos (estándares normativos con dimensión de peso y contenido moral). En su respuesta, Carrió ofrece elementos para afirmar que, a pesar de lo que cree Dworkin, la regla de reconocimiento puede incorporar, contingentemente, esos principios ${ }^{4}$. En cambio, de otro lado, Bulygin defiende una versión excluyente del positivismo jurídico, si bien algo alejada de aquella que suele ser atribuida a Joseph Raz. En efecto, según Bulygin, dada la controversia acerca de la existencia de hechos morales, ha de quedar abierta, con inclinación por la negativa, la posibilidad de que juicios morales incidan en la identificación de las fuentes del derecho ${ }^{5}$.

Sobresale también el tratamiento de Garzón Valdés y Nino como dos defensores del neoconstitucionalismo. Al respecto, cabe señalar

4 Carrió, G., Principios jurídicos y positivismo jurídico, Buenos Aires, Abeledo Perrot, 1970. 5 Véase Bulygin, E., El positivismo jurídico, Mexico, Fontamara, 2006. 
que el tono del volumen es mayoritariamente descriptivo, por lo que, salvo la mención algo imprecisa a que Garzón Valdés fue diplomático durante la dictadura, cuando en realidad tuvo que exiliarse y abandonar ese puesto una vez producido el golpe de Estado $^{6}$, no cabe mucho que cuestionar al trabajo de reconstrucción llevado adelante por los autores. Sin embargo, hay algunas afirmaciones en los textos de Barberis y Bongiovanni que abren espacio a consideraciones críticas, en particular, aquellas referidas a Garzón Valdés y Nino.

Tal como indican Barberis y Bongiovanni, Garzón Valdés defiende la tesis según la cual el punto de vista interno o del participante, tal como lo presenta Hart, debe ser concebido como un punto de vista moral. Es decir, según Garzón Valdés, la comprensión del derecho exige asumir el punto de vista de quién ve en la práctica jurídica una justificación de las obligaciones impuestas por el ordenamiento. Este modo de concebir el punto de vista interno y su incidencia en la comprensión del derecho, implicaría negar la tesis de la separabilidad entre derecho y moral, pues identificar el derecho tal como lo haces los participantes, exigiría involucrarse en las mismas consideraciones morales que ellos llevan a cabo. Contra estas afirmaciones, los autores sostienen que Garzón Valdés se equivoca, ya que Hart claramente señala que su teoría es descriptiva y que el punto de vista interno no implica aceptación moral del derecho. Dicho con otras palabras, según Hart, comprender, conocer y aplicar el derecho no exige aceptarlo desde un punto de vista moral, es posible conocer y aplicar, sin aceptar moralmente. En términos de la clasificación de Bobbio, Barberis y Bongiovanni dirían que Hart es un positivista metodológico, según el cual el conocimiento del derecho exige sólo un procedimiento descriptivo, y que Garzón Valdez lo convierte equivocadamente en un positivista ideológico. Sin embargo, me parece que esta es una reconstrucción,

6 Lo dice con precision Atienza: "Garzón Valdés was a diplomat in Germany when the 1976 coup d'etétat took place. He was removed from service and stayed in Germany", pág. 831 del Tomo 1. En cambio, la mención que hacen Barberis y Bongiovanni es algo imprecisa: "He started out as a diplomat under the military dictatorship in Argentina, was then exiled to Spain, and was finally awarded a professorship in Germany" pág. 265 del Tomo 2. 
cuanto menos, poco caritativa de la crítica de Garzón Valdés. Creo que autores como él buscan señalar la dificultad de estas afirmaciones explícitas de Hart. Es decir, aun cuando leyendo el concepto de derecho se pueda advertir claramente la pretensión descriptiva de Hart, el problema es que su concepción del punto de vista interno parece conducirlo, según Garzón Valdés, a sostener que quien acepta el derecho lo ha de hacer por razones morales, ello en virtud de la insistencia en el carácter práctico del punto de vista interno. Por ello, una respuesta a Garzón Valdez exige demostrar que tales consecuencias no se siguen de la teoría explícitamente propuesta por Hart.

Otro punto interesante es el análisis del argumento de Nino sobre el concepto de derecho. Como se sabe, en su libro póstumo Nino advertía ya el lugar central que la cuestión metodológica adquiriría unos años más tarde en el ámbito de la filosofía analítica del derecho. En ese libro, Nino expuso su posición, si bien creo todavía no completamente elaborada, según la cual el concepto de derecho, como cualquier otro concepto (con la exclusión de los pocos conceptos primitivos o de clase natural), es convencional, es decir, su contenido depende de nuestros acuerdos acerca de su uso y, por lo tanto, la inacabable discusión entre los filósofos acerca de las relaciones entre derecho y moral no es más que, o bien un desacuerdo meramente terminológico entre personas que usan diferentes criterios de uso del concepto, o bien una negociación para llegar a un acuerdo acerca de cuál es el mejor concepto que deberíamos usar para lograr ciertos fines. Barberis acepta esta "disolución" del problema propuesta por Nino. Sin embargo, de nuevo, me parece que tanto Nino como Barberis erran aquí la naturaleza del problema filosófico en juego cuando se discute acerca de la naturaleza del concepto de derecho. La discusión en esos casos puede ciertamente ser una discusión acerca de cuál es el concepto de derecho que mejor se adecua a ciertos fines metodológicos o académicos, pero esa no es la única discusión acerca de ese concepto. Muchas veces la discusión se refiere a cuál es el concepto de derecho en uso, principalmente, cuál es el concepto de derecho que puede inferirse a partir del discurso de los participantes en la práctica jurídica. Y esa respuesta no puede ser respondida por mera convención, es decir, no basta con que los 
filósofos del derecho se pongan de acuerdo acerca de cómo usarán el concepto de derecho para que ello ipso facto constituya el concepto de derecho usado por una comunidad. La identificación del concepto en uso exige proponer un método para reconstruir la práctica que, a su vez, depende de una propuesta teórica acerca de la naturaleza misma del concepto de derecho (convencional, primitivo, de clase natural, interpretativo, etc.). Dicho con otras palabras, si el concepto de derecho fuera convencional, entonces la "disolución" propuesta por Nino, y apoyada por Barberis, podría funcionar; pero el punto bajo discusión es precisamente si el derecho puede o no ser concebido como un concepto convencional, i.e., un concepto cuyos criterios de uso dependen de un acuerdo (o convergencia) entre los participantes de la práctica. Éste último es, creo, uno de los puntos centrales de la disputa entre iusnaturalismo y iuspositivismo tal como se ha estructurado en la última mitad del siglo 20, i.e., la identificación de la naturaleza del concepto de derecho y del contenido de nuestro concepto de derecho. El partiacque entre iuspositivismo e iusnaturalismo queda marcado aquí según se sostenga o no que para responder a ambas preguntas es necesario involucrarse en consideraciones morales.

Finalmente, Chiassoni se refiere con más de detalle a la teoría de Bulygin sobre la interpretación. Bulygin sostiene, tal como señala Chiassoni, un punto de vista intermedio, similar al hartiano, entre el escepticismo y el formalismo. Sin embargo, su teoría se caracteriza por distinguir, a diferencia de la posición intermedia de Hart, entre interpretación en abstracto e interpretación en concreto. La primera está dirigida a normas, es decir, a traducir formulaciones de normas en normas, y la segunda está dirigida a casos, es decir, a subsumir casos individuales en casos genéricos, que la norma, previamente identificada por la interpretación en abstracto, conecta con una consecuencia normativa. Según Bulygin, en ambas instancias la interpretación puede ser tanto una cuestión de descubrimiento como una cuestión de decisión. Es decir, a veces una formulación normativa es ambigua y, por lo tanto, el intérprete tiene que decidir cuál de todos los significados asociar a la formulación, y otras veces cuál sea el significado es materia de descubrimiento, o bien porque la formulación posee uno solo o bien 
porque el contexto permite identificarlo. Del mismo modo, la subsunción de un caso puede verse afectada por la vaguedad de la norma y, en esas situaciones, de nuevo, el intérprete deberá decidir si incluir o no el caso individual en el caso genérico. Pero junto a casos indeterminados hay también casos claros de inclusión y de exclusión. La diferencia es que, mientras en el caso de la interpretación en abstracto la ambigüedad es un dato de hecho, i.e. depende de los usuarios del lenguaje si un término está o no asociado a más significados, en el caso de la interpretación en concreto, la vaguedad es un rasgo ineliminable del lenguaje que usa predicados. Chiassoni critica este modo de ver de Bulygin sosteniendo, primero, que el uso del lenguaje es bien distinto del señalado por Bulygin y, segundo, que en el ámbito jurídico el tipo de uso que se hace del lenguaje está ligado esencialmente a disputas de intereses donde el supuesto significado literal de las palabras utilizadas en las formulaciones normativas es sólo una de las múltiples opciones que tienen a disposición los intérpretes. En otras palabras, el problema con la teoría intermedia de Bulygin es que aquello que en el ámbito del lenguaje ordinario puede ser visto como un acto de descubrimiento del único significado (literal) de un término, en ámbito jurídico constituye una elección, por parte del intérprete, de uno entre varios significados disponibles. Si bien comparto en líneas generales las observaciones de Chiassoni, me parece que las críticas a Bulygin no deben oscurecer la centralidad del significado literal para la interpretación jurídica, como punto de partida indispensable para la comprensión de las disposiciones normativas. Ese reconocimiento no define la disputa entre formalismo, teoría intermedia y escepticismo, pues, desde mi punto de vista, la tesis central del escepticismo no es tanto acerca de la ontología de los significados de las disposiciones normativas, sino acerca de los usos que de ellas hacen los operadores jurídicos.

\section{Palabras de cierre}

$\mathrm{Al}$ inicio ya se mencionó que el Volumen 12 aquí reseñado es la conclusión del proyecto iniciado hace ya más de una década por Enrico 
Pattaro, actualmente acompañado por Corrado Roversi, titulado $A$ Treatise of Legal Philosophy and General Jurisprudence. En estas breves líneas he intentado mostrar que la organización de los dos Tomos que lo conforman, basada en áreas geográficas y lingüísticas, orientaciones teóricas y temas permite construir una suerte de trama para comprender la contribución de la cultura del civil law a la filosofía del derecho durante el siglo 20. Aquí me he limitado a presentar sucintamente cada capítulo; he hecho algunas referencias y observaciones críticas un poco más extensas respecto del tratamiento dado a la filosofía del derecho en Latinoamérica, concentrándome, sobre el final, en algunos autores argentinos. La finalidad de la reseña es pues introducir en la agenda de lectura esta obra, de consulta indispensable, junto con el resto del Treatise, para cualquier intento de acercarse a la historia de la filosofía del derecho.

\section{Bibliografía}

Atienza, M., La filosofía del derecho argentina actual, Buenos Aires, Depalma, 1984.

Benavente, J.W., "La filosofía del derecho en Chile", Anuario de Filosofia Jurídica y Social, 1, 1983, pp. 21-39.

Bulygin, E., El positivismo jurídico, Mexico, Fontamara, 2006.

Carrió, G., Principios jurídicos y positivismo jurídico, Buenos Aires, Abeledo Perrot, 1970.

Evald, J., Alf Ross - a life, Tilst, DJOF, 2014. 